\title{
Representaciones sociales del pasado: el golpe de Estado del 11 de septiembre de 1973 según niños, niñas y adolescentes de la comuna de la Ligua, región de Valparaíso-Chile ${ }^{1}$
}

\author{
Ximena Faúndez Abarca ${ }^{2}$, Fuad Hatibovic Díaz ${ }^{3}$, Manuel Cárdenas Castro 4 , \\ Juan Sandoval Moya ${ }^{5}$, Romina Adaos Orrego ${ }^{6}$ y Verónica Rodríguez Espinoza ${ }^{7}$ \\ Universidad de Valparaiso-Chile
}

Se exploraron representaciones sociales del 11 de septiembre de 1973 — fecha del golpe de Estado en Chile- en una muestra de 1053 nińos, niñas y adolescentes, 550 mujeres y 503 hombres, de liceos municipales y subvencionados de la comuna de la Ligua, región de Valparaíso. Los participantes tenían entre 11 y 18 años de edad $(M=14.83 ; D E=1.89)$. Se utilizó el Cuestionario de Diagnóstico Participativo en Infancia y Adolescencia. Los datos se analizaron en dos etapas, primero se construyeron diccionarios de palabras, posteriormente se realizaron análisis de conglomerados jerárquicos, escalamiento multidimensional y correspondencias múltiples. Aproximadamente la mitad de los participantes no logró asociar conceptos a la fecha o asoció conceptos relativos a otros eventos históricos. Entre quienes sí relacionaron conceptos, mencionan consecuencias negativas a nivel social y personal, tales como violación a los Derechos Humanos, muerte, sufrimiento y violencia política, lo cual se conjuga con la creencia de una violencia fundacional que habría posibilitado progreso en Chile.

Palabras clave: representaciones sociales, memoria, golpe de Estado, infancia, adolescencia.

Social Representations of the Past: The Coup of September 11, 1973 according to children and adolescents from la Ligua commune in Valparaiso, Chile

This paper analyzes the social representations of September 11, 1973, the date of the coup in Chile, of a sample of 1053 boys, girls and adolescents from Valparaíso. Participants ranged in age between 11 and $18(M=14.83$; $S D=1.89)$. The Participatory Diagnostic Questionnaire in Childhood and Adolescence was used. The data were analyzed in two phases;

1 Este artículo contó con apoyo del Centro de Estudios Interdisciplinario sobre Cultura Política, Memoria y Derechos Humanos de la Universidad de Valparaíso y del Proyecto CONICYT PIA/ANILLO SOC180007

2 Doctora en Psicología (Pontificia Universidad Católica de Chile). Profesora adjunta de la Escuela de Psicología de la Universidad de Valparaíso y miembro del claustro del Doctorado en Estudios Interdisciplinarios sobre Pensamiento, Cultura y Sociedad de la misma Universidad. Directora del Centro de Estudios Interdisciplinarios sobre Cultura Política, Memoria y Derechos Humanos (CEI-CPMDH) de la Universidad de Valparaíso. Dirección postal: Hontaneda 2653 Valparaíso-Chile. Contacto: ximena.faundez@uv.cl. https://orcid. org/0000-0003-1987-7905

3 Doctor en Psicología Social (Universidad del País Vasco). Profesor adjunto de la Escuela de Psicología de la Universidad de Valparaíso. Investigador del CEI-CPMDH de la Universidad de Valparaíso. Dirección postal: Hontaneda 2653 Valparaíso-Chile. Contacto: fuad. hatibovic@uv.cl. https://orcid.org/0000-0002-5144-3341 
first dictionaries were constructed, and then analyses of hierarchical clusters, multidimensional scaling and multiple correspondences were performed. Almost half of the participants failed to associate concepts with the date or associated concepts to other historical events. For those who did relate the concepts, they discuss negative consequences at a social and personal level, such as human rights violations, death, suffering and political violence. This is combined with the belief of a foundational violence that would have made social progress possible.

Keywords: Social representations, memory, coup, childhood and adolescence.

\section{Representaçóes sociais do passado: 11 de setembro de 1973 de acordo com crianças e adolescentes}

O presente estudo explorou as representaçôes sociais de 11 de setembro de 1973 - golpe de Estado no Chile - de uma amostra de 1053 meninos, meninas e adolescentes de Valparaíso. Os participantes tinham entre 11 e 18 anos de idade $(\mathrm{M}=14.83$ e $\mathrm{SD}=1.89)$. Foi utilizado o questionário de diagnóstico participativo na infância e na adolescência. A análise de dados foi realizada em duas etapas, os primeiros dicionários foram construídos com palavras de conteúdo semelhante (Di Giacomo, 1987), análise posterior de conglomerados hierárquicos, escalamento multidimensional e correspondências múltiplas foram realizadas. Entre os resultados, nota-se que quase metade dos participantes náo associam conceitos à data ou associam conceitos relacionados a outros eventos históricos. Entre aqueles que relacionam conceitos, mencionam consequências negativas a nível social e pessoal, o que é combinado com a crença de uma violência fundamental que teria possibilitado o progresso social no Chile.

Palavras-chave: representaçôes sociais, memória, golpe de Estado, infância e adolescência.

4 Doctor en Comportamiento Social y Organizacional (Universidad Autónoma de Madrid). Profesor titular de la Escuela de Psicología de la Universidad de Valparaíso. Dirección postal: Hontaneda 2653 Valparaíso-Chile. Contacto: manuel.cardenas@uv.cl. https://orcid. org/0000-0002-5484-0078

5 Doctor en Psicología Social (Universidad Complutense de Madrid). Profesor titular de la Escuela de Psicología de la Universidad de Valparaíso y miembro del claustro del Doctorado en Estudios Interdisciplinarios de esta misma Universidad. Es investigador del CEI-CPMDH) de la Universidad de Valparaíso. Dirección postal: Hontaneda 2653 Valparaíso-Chile. Contacto: juan.sandoval@uv.cl. https://orcid.org/0000-0003-4648-2757

6 Psicóloga (Universidad Católica del Norte), Doctoranda en Estudios Interdisciplinarios sobre Pensamiento, Cultura y Sociedad (Universidad de Valparaíso). Dirección postal: Hontaneda 2653 Valparaíso-Chile. Contacto: romina.adaos@gmail.com

7 Magíster en Educación con mención en Gestión Educativa (Universidad de las Artes y Ciencias Sociales). Coordinadora Centro de Asesoría Psicoeducativa en la Escuela de Psicología de la Universidad de Valparaíso. Dirección postal: Hontaneda 2653 Valparaíso-Chile. Contacto: veronica.rodríguez@uv.cl. https://orcid.org/0000-0002-5433-794X 
Représentations sociales du passé : coup d'État du 11 septembre 1973 selon les enfants et les adolescents de la commune de La Ligua, région de Valparaiso-Chili.

Cette étude a exploré les représentations sociales du 11 Septembre, 1973 - coup d'Etat au Chili d'un échantillon de 1053 enfants et adolescents de Valparaíso. Les participants avaient entre 11 et 18 ans $(M=14.83 ; S D=1.89)$. Le questionnaire de diagnostic participatif sur l'enfance et l'adolescence a été utilisé. L'analyse des données a été réalisée en deux étapes, le dictionnaire de contenu similaire, puis une analyse typologique, mise à l'échelle multidimensionnelle et des correspondances multiples ont été effectuées est d'abord construit. Parmi les résultats, il convient de noter que près de la moitié des participants n'associent pas les concepts à la date ou associent des concepts liés à d'autres événements historiques. Parmi ceux qui se rapportent concepts mentionnés niveau personnel et social négatif, qui est associé à une croyance fondamentale de la violence qui aurait permis le progrès social au Chili.

Mots-clés: représentations sociales, mémoire, coup d'Etat, enfance et adolescence. 
Para Halbwachs (1925), la memoria se construye a través de marcos sociales, los que entiende como instrumentos que permiten articular los recuerdos, dotándolos de sentido. El tiempo es uno de estos marcos. Así, la memoria del pasado se construye y reconstruye en el presente, motivada por objetivos actuales y con vistas al futuro (Halbwachs, 1950). Decir que la memoria se construye y reconstruye significa que recordar es un proceso activo y dinámico. Por lo tanto, es posible afirmar que la memoria colectiva constituye un conjunto de representaciones compartidas sobre eventos relevantes del pasado y que un determinado grupo produce y transmite por medio de la interacción informal entre sus miembros, con vistas a su utilización en el presente (Moscovici, 1979; Jodelet, 2012). Estas representaciones tienen una finalidad práctica: delimitar la identidad del grupo, resaltar ciertos aspectos de su genealogía y orientar las acciones de los miembros del grupo respecto de los otros grupos presentes en el campo social (Le Paumier \& Zavalloni, 2002). Los eventos representados constituyen una narrativa específica sobre el pasado, que permite comunicar significados y sirve como guía para la acción presente (Bobowik et al., 2014; Liu \& Hilton, 2005).

Es probable que aquellos eventos que han significado un profundo cambio cultural sirvan como base para la creación de representaciones, dado que permiten mantener la cohesión del grupo mediante procesos de identificación, así como justificar sus conductas presentes sobre la base de ciertos valores que las figuras asociadas a dicho pasado representan. Del mismo modo, dado que estos eventos suelen implicar un quiebre entre grupos, dispondrán de diversas representaciones sobre el evento pasado en cuestión, las que se mantendrán en disputa. La multiplicidad de representaciones del pasado en disputa dará cuenta de la diversidad social de quienes las elaboran (Doise, 1991), así como de las fuertes reacciones emocionales que dicho pasado suscita (Faúndez \& Azcárraga, 2014). 
El carácter constructivo de dichas representaciones del pasado implica que el contenido de las mismas pueda variar respondiendo a las necesidades e intereses del grupo en el presente. Se trataría de una construcción social, de carácter contingente y contextual (Middleton \& Edwards, 1990) que respondería a las necesidades de dominio material y simbólico de un grupo social. En este sentido, las representaciones sociales sobre eventos del pasado nos informan más sobre quiénes las elaboran que de los hechos acaecidos, donde se ajusta la sabiduría del pasado a la luz de los desafíos del presente y futuro (Liu \& Sibley, 2009), siendo las condiciones presentes en que se encuentra un determinado grupo las que constrińen las posibilidades del recuerdo, en tanto funcionan como marcos sociales, de ahí su relación con la memoria social (Banchs, 2000). De este modo, la representación social es el proceso psicosocial por el que opera la construcción del recuerdo de un evento u objeto social particular (de Alba, 2016; Haas \& Jodelet, 1999), no necesariamente experimentado personalmente, y que son transmitidas y conservadas por un grupo a través de la comunicación de sus miembros (Páez, Bobowik, Liu \& Basabe, 2016).

Estas representaciones sobre el pasado suelen girar en torno a hechos emblemáticos, ya sea porque dividen a los diferentes grupos y generan procesos identitarios diferenciadores alrededor de ellos (memorias en disputa) o porque permiten, por el contrario, generar efectos de cohesión social (Joya, 2006).

$\mathrm{Al}$ igual que cualquier representación social, las representaciones del pasado incluyen una dimensión informativa sobre dichos hechos, una actitud más o menos favorable y un campo de representación (Jodelet, 1987; Moscovici, 1979). La información es generada de forma activa por los grupos, exponiéndose a ciertos medios que le son afines y atendiendo a ciertos aspectos de la realidad analizada. La información variará en cantidad y calidad dependiendo de la importancia relativa atribuida al fenómeno y la ubicación social del grupo que la genera o recopila. La actitud refiere a una orientación global asociada al objeto de representación, que puede ser más o menos positiva y que expresa la orientación evaluativa respecto del mismo, pudiendo dinamizar 
conductas y generar reacciones emocionales en los integrantes del grupo. El campo de representación implica la creación de una imagen o modelo social basado en la integración y organización de la información (Sandoval, 2004). Este se establece a partir de las cualidades de las unidades aprehendidas, unas posibilitan concentrar imágenes y significados capaces de dar vida al objeto representado, y otras facilitan reavivar aspectos concretos; en su conjunto conforman una red de elementos tejidos por combinaciones de unas y otras. El campo de la representación se organiza en torno al núcleo figurativo. Este sería un conjunto de imágenes con forma gráfica y coherente que encierra la esencia del concepto. Permite comprender las cosas de forma más sencilla y a través de su uso, se convierte en un hecho natural. El núcleo figurativo organiza los elementos de la representación y es la parte más sólida y estable de esta. De él depende el significado global de la representación (Knapp, Suárez \& Mesa, 2003).

Los elementos centrales, es decir, aquellos más estables y compartidos por el grupo son los que definen la organización de toda la representación, manifestando la memoria compartida del grupo; mientras que aquellos más anecdóticos y dinámicos se ubican como elementos perífericos de la representación (Abric, 1994). En el presente trabajo se optó estudiar el campo de representación ya que este constituye la organización del conjunto de conceptos que un grupo social asocia con mayor fuerza al objeto de representación.

En Chile, uno de estos eventos del pasado reciente que implicó un quiebre institucional y social relevante, y que sigue suscitando fuertes reacciones emocionales y disputas por su representación, es el golpe de Estado de 1973 y la posterior dictadura cívico-militar. La mayor parte de los estudios han abordado el fenómeno del recuerdo de dicha fecha, lo han hecho trabajando exclusivamente con población adulta de la región Metropolitana (Manzi, 1999; Manzi, Helsper, Ruiz, Krause \& Kronmüller, 2003). Aun cuando varios trabajos han enfatizado la relevancia cotidiana del recuerdo de dichos acontecimientos a nivel familiar (Contreras, Grass \& Nicholls, 2009) y de los procesos de transmisión y dialogía inter y transgeneracional entre familiares 
de víctimas de la dictadura (Faúndez, Brackelaire \& Cornejo, 2013; Reyes, Cornejo, Cruz, Carrillo \& Caviedes, 2015). Estudios en población general, plantean que existe un menor grado de información sobre dichas fechas entre los jóvenes chilenos, pero un expresivo deseo por hablar sobre las mismas (Arnoso, Cárdenas \& Páez, 2012; Fundación OPCION, 2013).

Los estudios sobre memorias de la dictadura militar de estudiantes chilenos se han realizado casi exclusivamente en escuelas secundarias (Veneros y Toledo, 2009; Castro, 2013; Vásquez, 2014), de las ciudades de la zona centro del país, por lo que no se cuenta con información de la gestión curricular de estos contenidos en escuelas primarias de regiones extremas ni de zonas rurales. Las investigaciones revisadas dan cuenta que el desafío de incorporar la historia reciente y la educación en derechos humanos, es asumido de modo parcial por la institución escolar. Magendzo y Toledo (2009) indican que los docentes, reconocen que en su mayoría no pasaron la unidad relativa a la historia reciente de Chile, aludiendo a la falta de tiempo, la inexistencia de la planificación de la unidad y la carencia de fuentes documentales y material didáctico.

La teoría de las representaciones sociales resulta pertinente para analizar las memorias sociales del pasado reciente en la medida que comparten importantes supuestos, tales como su concepción constructivista de la realidad social que hace que los recuerdos sean considerados como una construcción colectiva de un relato respecto a los hechos del pasado. En ningún caso la representación es fija y, como en los procesos de evocación de memorias, el contexto de evocación de la representación posee una enorme relevancia, así como los procesos colectivos de comunicación e interacción intra e intergrupal. En este sentido dichas teorías resultarían complementarias, no pudiendo entenderse la memoria colectiva sin la alusión a un conjunto de representaciones que la sostienen en el presente (Haas \& Jodelet, 1999). Del mismo modo, el concepto de memoria social aporta una dimensión temporal e histórica ausente de las principales formulaciones de la teoría de las representaciones sociales y que permite la confluencia de pasado, presente y futuro en la construcción social de la realidad (De Alba, 2016). 
En este sentido, no es posible representarse el presente sin alusión a los recuerdos del pasado (en tanto funcionarían como conocimiento sobre el pasado) y tampoco sería posible sostener el recuerdo sino como soporte de una identidad presente y en vistas a la construcción de unas representaciones de lo actual.

El presente estudio tuvo por objetivo explorar las representaciones sobre el 11 de septiembre de 1973 — fecha del golpe de Estado en Chile_-, a nivel del campo de representación, en NNA, estudiantes de educación primaria y secundaria, de la comuna de La Ligua, región de Valparaíso. En el entendido que estos se apropian de dicho pasado y lo representan incorporándolo para fines identitarios (Faúndez et al., 2013) y que estas representaciones delimitan en buena medida sus formas de socialización política presente (Davoine \& Gaudilliere, 2011) sobre la base de la cercanía familiar a los hechos (Carvacho, Manzi, Haye, González \& Cornejo, 2013; Palma, 2015; 2017). Intentaremos observar cuáles son las representaciones sobre el 11 de septiembre de 1973 — fecha del golpe de Estado_-, así como las dimensiones subyacentes que organizan dichas representaciones.

\section{Estudios de memoria de la dictadura e infancia y juventud}

La mayor parte de los estudios sobre representaciones sociales del pasado traumático (Arnoso et al., 2015; Manzi, 1999; Manzi, Helsper, Ruiz, Krause \& Kronmüller, 2003) se han centrado en población adulta. Esto puede relacionarse con el hecho de que las etapas de desarrollo previas a la adultez han sido consideradas históricamente como desprovistas de politicidad, como un momento colonizado por la racionalidad adulta sobre la niñez, siendo niños y niñas identificados como "preciudadanos" (Fundación OPCION, 2013; Manzi, 1999) por lo tanto, el estatus de sujeto de derechos como tal aún no es conferido por la sociedad adulta.

En este sentido, no es extraño que los estudios que abordan la posición de niños, niñas y adolescentes (en adelante NNA) frente a los acontecimientos políticos ocurridos en el pasado reciente de nuestro país sean escasos. Este hecho resulta significativo puesto que las generaciones 
actuales de NNA de nuestro país forman parte de procesos de recepción y transmisión de carácter transgeneracional de los sucesos de violencia política vividos en Chile (Faúndez, Cornejo \& Brackelaire, 2014).

Se ha señalado que los jóvenes chilenos poseen menos información que los adultos sobre las comisiones de Verdad y Reconciliación y que, por el contrario, los deseos de hablar sobre el pasado son más altos en estos que en la población de mayor edad (Arnoso et al., 2012). Sin embargo, cuando los jóvenes son descendientes (hijos o nietos) de víctimas de la dictadura cívico-militar, se ha encontrado que estos poseen un alto nivel de conocimiento de la dictadura y del trauma vivido por sus ascendientes. De esta forma, los jóvenes se sitúan como herederos del pasado familiar traumático, apropiándose de este e incorporándolo en su identidad social y política (Faúndez et al., 2013).

Por otra parte, respecto al modo en que se han percibido las políticas de reparación, se han encontrado diferencias. Se plantea que haber vivido o no durante la época de la dictadura, la posición política y la cercanía a hechos de violencia política son factores que determinarían el contenido de la memoria histórica de la ciudadanía (Carvacho et al., 2013; Reyes, 2007, 2015).

El conocimiento histórico de NNA sobre los hechos acaecidos desde el golpe de Estado hasta el fin de la dictadura, varía en contenido y profundidad; siendo los elementos más mencionados en sus respuestas: Salvador Allende, golpe de Estado, Augusto Pinochet y toque de queda. Los estudiantes de sexto ańo básico cuando relatan el golpe de Estado y la muerte de Allende sitúan estos hitos como el inicio de una época. Así, demuestran que poseen un relato ordenado de los hechos en términos cronológicos (Palma, 2015).

Un estudio con NNA de entre 10 y 17 ańos realizado a cuarenta años del golpe de Estado en Chile, plantea que entre los personajes históricos más relevados por los NNA se encuentran Bernardo O’Higgins (51.9\%) y Arturo Prat (47\%). Personajes secundarios para los NNA resultan ser Augusto Pinochet (8.6\%) y Salvador Allende (7.8\%) sugiriendo el escaso significado que reviste para ellos el periodo histórico que estos actores representan (Fundación OPCIÓN, 2013). Se ha observado 
que NNA si bien identifican el hito del golpe de Estado y sus principales protagonistas, no lo asocian a un proceso histórico de más largo alcance temporal o espacial y tampoco incorporan la dimensión política a su comprensión (Díaz, Faivovich, Carrasco, Glavic \& Vivanco, 2013).

Los NNA conocen sobre el pasado glorioso o doloroso de sus naciones a través de la trasmisión familiar, la instrucción escolar formal y la exposición al tratamiento que los medios de comunicación hacen sobre el tema (Palma, 2017). Para la mayoría de los/as NNA sus principales referentes de memoria sobre el pasado reciente de Chile son sus familiares (Davoine \& Gaudilliere, 2011; Faúndez et al., 2013). Por otro lado, se ha estudiado la enseñanza-aprendizaje de la dictadura como una unidad perteneciente al currículum de historia y geografía de algunas escuelas (Rojas \& Vargas, 2013), y la vivencia infantil del clima de violencia en dictadura en un grupo de jóvenes de la ciudad de Santiago (Magendzo, Rubio \& Aubel, 1999).

Cuando se han abordado los temas "controversiales" en la Escuela, como las violaciones de derechos humanos, se ha encontrado que no existe relación entre nivel de controversia y su enseñanza. En este caso, los estudiantes asumen un rol activo, valoran el diálogo, solicitan la opinión del profesor y desean construir sus interpretaciones sobre la historia (Toledo, Magendzo, Gutierrez, Iglesias \& López-Facal, 2015). También se ha encontrado en estos espacios educativos, la necesidad de los estudiantes de conocer y expresar sus memorias, existiendo una tensión en la integración de su historia familiar con la colectiva. En estos casos se observan tensiones y conflictos entre la memoria familiar y la memoria colectiva (Toledo \& Magendzo, 2013). Y cuando los estudiantes han tenido la oportunidad de aproximarse a un relato directo de un/a sobreviviente de la dictadura, muestran emociones de solidaridad y de contacto fraterno entre sus pares (Toledo et al., 2015).

La pregunta de investigación que guió este estudio fue la siguiente: ¿Cuáles son las representaciones sociales, a nivel del campo de representación, sobre el 11 de septiembre de 1973 (fecha del golpe de Estado en Chile) de estudiantes de primaria y secundaria de la comuna de La Ligua, región de Valparaíso? 


\section{Método}

\section{Participantes}

La muestra quedó conformada por 1053 participantes, 550 mujeres (52.2\%) y 503 hombres (47.8\%), pertenecientes a liceos municipales y subvencionados de la comuna de la Ligua, región de Valparaíso (Chile), y cuyas edades fluctuaron entre 11 y 18 ańos $(M=14.83$ y $D E=1.89)$. La Ligua es una comuna ubicada en la región de Valparaíso, capital de la provincia de Petorca. Se ubica $154 \mathrm{~km}$ al norte de Santiago, capital de Chile, y $110 \mathrm{~km}$ al norte de Valparaíso, capital regional. La Ligua se caracteriza por tener un alto porcentaje de población que habita en zonas rurales.

Los niveles educacionales cursados por los adolescentes incluidos en la muestra fueron séptimo básico (19.6\%), octavo básico (10.7\%), primero medio $(20.8 \%)$, segundo medio $(15 \%)$, tercero medio $(17.8 \%)$ y cuarto medio (16\%). El 12\% de los estudiantes asistía a colegios ubicados en zonas rurales y un $88 \%$ lo hacía en colegios de zonas urbanas.

Cabe señalar que en Chile, durante la dictadura cívico-militar, se realizó una reforma educacional que trasladó y descentralizó la administración de las escuelas del sistema público central, al sistema municipal. Se creó un subsidio estatal que no distinguió si la educación era provista por escuelas privadas que cobraran a los alumnos o por escuelas municipales gratuitas, generándose así dos tipos de escuelas subvencionadas: escuelas municipales, cuya administración estaba a cargo de la municipalidad correspondiente, y escuelas particulares subvencionadas, dirigidas por privados. Ambas obtenía subvención por alumno y por su asistencia, con el objetivo de incentivar la competencia entre las escuelas.

\section{Medición}

El Cuestionario de Diagnóstico Participativo en Infancia y Adolescencia incluyó siete dimensiones y un total de 25 ítemes. Las seis primeras dimensiones abordan los aspectos sociodemográficos, bienestar, 
apoyo social percibido y la percepción de los participantes respecto a la experiencia de ser NNA de la comuna de la Ligua. En el presente artículo analizamos los datos de la séptima dimensión, referida a representaciones sociales del pasado. Esta última dimensión incluía un ítem en que se presentaba a los participantes una palabra-estímulo a la que debían asociar libremente otras palabras. Dicho estímulo era la fecha del 11 de septiembre de 1973. El método de asociación libre de palabras trabaja sobre el supuesto que las personas tienen un limitado número de significados asociados a un determinado objeto social y que los contenidos asociados a la representación de dicho objeto se encuentran estructurados sobre la base de su semejanza o distancia con otros significados (Cárdenas, 2008, Doise, Clémence \& Lorenzi-Cioldi, 1993). Del mismo modo, se trabaja sobre el supuesto que la representación está orientada por pocas dimensiones actitudinales (Arnoso, Páez, Cárdenas, Zubieta, Espinosa \& Bilbao, 2015). Debido a que solo presentaremos los datos producidos por el método de asociación libre, es importante señalar que para este método no se aplican los procedimientos tradicionales de validación, como el cálculo de fiabilidad o el análisis factorial. El método de asociación libre ha sido ampliamente usado en estudios de Psicología Social para la medición de las representaciones sociales (Di Giacomo, 1980, 1987; Cárdenas \& Blanco, 2004; Cárdenas, 2008).

\section{Procedimiento}

El estudio surge como parte de un proceso de diagnóstico participativo en infancia y adolescencia, solicitado por la Oficina de Protección de Derechos (OPD) y la Red de Infancia de la comuna de La Ligua con el objetivo de contar con información que permitiera elaborar una política de infancia al interior de la comuna. El proyecto de investigación que permitió la obtención de datos fue revisado y aprobado en cuanto a los aspectos éticos por la Oficina de Protección de Derechos (OPD) de La Ligua.

El proceso de producción de información se llevó a cabo entre los meses de junio y agosto del año 2016. El contacto y reclutamiento de los participantes se realizó, mediante los directores de los establecimientos 
educacionales a los cuales pertenecen los participantes de este estudio. Previo al levantantamiento de información con NNA se realizó un proceso de consentimiento informado con los directores de establecimientos educacionales. Posteriormente se realizó este mismo proceso con los padres y/o tutores responsables de los/as NNA. Finalmente se realizó un proceso de asentimiento con los participantes. Todos los participantes de este estudio fueron informados sobre los objetivos y procedimientos de la evaluación, asegurándoseles la confidencialidad de la información. Los/as NNA que participaron del proceso fueron aquellos que contaban con el documento firmado por el padre y/o tutor, el cual fue entregado al equipo investigador y que asintieron participar.

Cada participante respondió un cuestionario compuesto por $25 \mathrm{de}$ ítems en sesiones de entre 30 y 45 minutos en la sala de clases. Los cuestionarios fueron aplicados por profesionales capacitados para esta tarea.

\section{Análisis de datos}

El análisis de los datos se realizó a partir de una planilla construida sobre la base de las palabras asociadas a los estímulos, las cuales son organizadas en torno a diccionarios que cumplen la función de ordenar y condesar aquellas de similar contenido (Di Giacomo, 1987; Cárdenas, 2008). Para los análisis posteriores se han seleccionado las palabras con una mayor frecuencia de asociaciones. De este modo, la producción total de palabras ascendió a 1250 términos, lo que equivale a un promedio 2.3 términos por persona. Un total de 517 personas no asocian concepto alguno al estímulo "11 de septiembre de 1973". De este total se ha reducido la cifra a los 12 términos de mayor frecuencia y que constituyen el diccionario definitivo (todas aquellas con frecuencia > 15). Los 12 términos en conjunto contienen 593 palabras asociadas. Sobre la base de este diccionario se ha construido una matriz binaria (presencias o ausencias), la que se ha utilizado para crear una matriz de distancias a partir de la cual se realiza el análisis de conglomerados jerárquicos, escalamiento multidimensional y análisis de correspondencias múltiples. Se utilizaron los programas SPSS 20.0 y STATISTICA 10.0. 


\section{Resultados}

A continuación se presentan los resultados de forma general para todos los participantes del estudio. Luego se analizan y comentan las particularidades asociadas a las diferencias de género (hombre o mujer) y edad/nivel de estudios (séptimo, octavo y primero medios versus segundo, tercero y cuarto medio) de los participantes. La segmentación por género se debe a que diversos estudios (Faúndez, Cárdenas, Hatibovic, Palma \& Bravo, 2017; Hiner, 2015; Jelin, 2002) han mostrado que existen diferencias en las memorias del pasado reciente de mujeres y hombres asociadas los roles sociales hegemónicos y a expectativas culturales asociadas al sistema sexo-género (Rubin, 1986). La segmentación por nivel de estudios, se debe a que diversos estudios (Faúndez et al., 2013; Carvacho, et al., 2013; Reyes, 2007, 2015) planean diferencias en las memorias del pasado reciente asociados a la edad de los participantes.

El primer antecedente relevante a tener en consideración es que casi la mitad de los participantes no logran asociar conceptos a la fecha señalada o asocian conceptos relativos a otros eventos históricos. Entre los otros eventos históricos nombrados figuran: el atentado de las Torres Gemelas del 11 de septiembre de 2001, en Estados Unidos o la Primera Junta Nacional de Gobierno de Chile el ańo 1810.

El análisis de conglomerados (Figura 1) nos informa la presencia de un agrupamiento que vincula el 11 de septiembre con la dictadura militar y la violación de derechos humanos, esto es, con las consecuencias asociadas a los acontecimientos acaecidos en dicha fecha. A este mismo agrupamiento se agrega la figura de Pinochet. Asociado a este personaje, un segundo conglomerado agrupa los términos muerte, sufrimiento y violencia política, los cuales refieren a consecuencias más específicas vinculadas a la fecha propuesta, un tercer conglomerado relaciona las palabras violencia y progreso. Una lectura general de la gráfica nos informa de la asociación entre el 11 de septiembre con las consecuencias negativas a nivel social y personal, pero también de la creencia de una violencia de carácter fundacional que habría posibilitado un 
cierto progreso social. Es decir, se reconocerían los elementos negativos del golpe de Estado y posterior dictadura, pero se les vincularía también con un discurso referido al progreso.

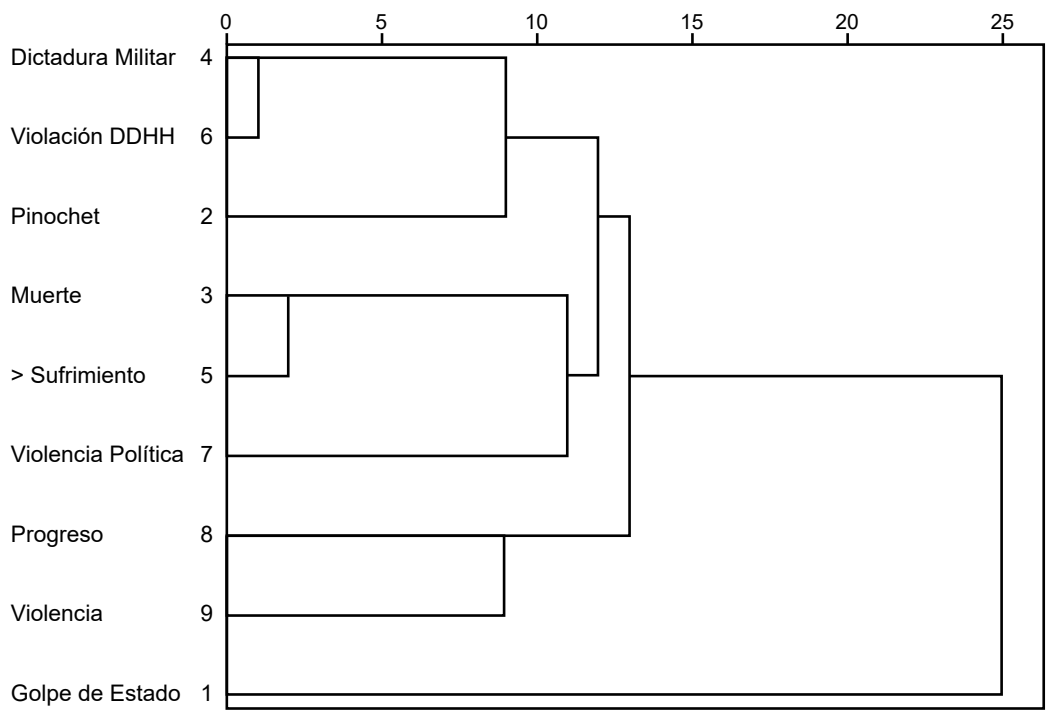

Figura 1. Análisis de conglomerados jerárquico al estímulo "11 de septiembre de 1973"

Los análisis realizados segmentando la muestra por sexo nos indican leves diferencias entre hombres y mujeres. El primer conglomerado muestra que los hombres vinculan las figuras históricas de Allende y Pinochet con el bombardeo al Palacio de la Moneda y que las mujeres vinculan estas figuras con el golpe de Estado. El primero, más centrado en la descripción del hecho y, el segundo, en el significado político del gesto. Un segundo conglomerado, equivalente para ambos grupos, asocia la dictadura militar con la violación de derechos humanos. El tercer clúster en ambos grupos vincula muerte, sufrimiento e injusticia. Sólo para el caso de los hombres la asociación entre violencia y progreso aparece como relevante. 
Del mismo modo al segmentar la muestra utilizando la variable nivel educacional, constatamos que aquellos jóvenes de menor edad (séptimo, octavo y primero medio) se representan la fecha a partir de sus consecuencias presentes, asociando fuertemente la dictadura, la violación de derechos humanos y la idea de progreso (aunque ciertamente esta última se agrega de modo bastante más tardío). Para los niveles más altos (segundo, tercero y cuarto medio), las figuras históricas de Pinochet y Allende se anudan al bombardeo del palacio de la Moneda.

Los índices de ajuste para el escalamiento multidimensional Stress (.066) y RSQ (.991) nos indican que nuestros datos son apropiados para realizar este tipo de análisis, logrando la convergencia adecuada. Los resultados nos indican la presencia de una primera dimensión (Figura 2) que opone el golpe de Estado con sufrimiento y muerte,

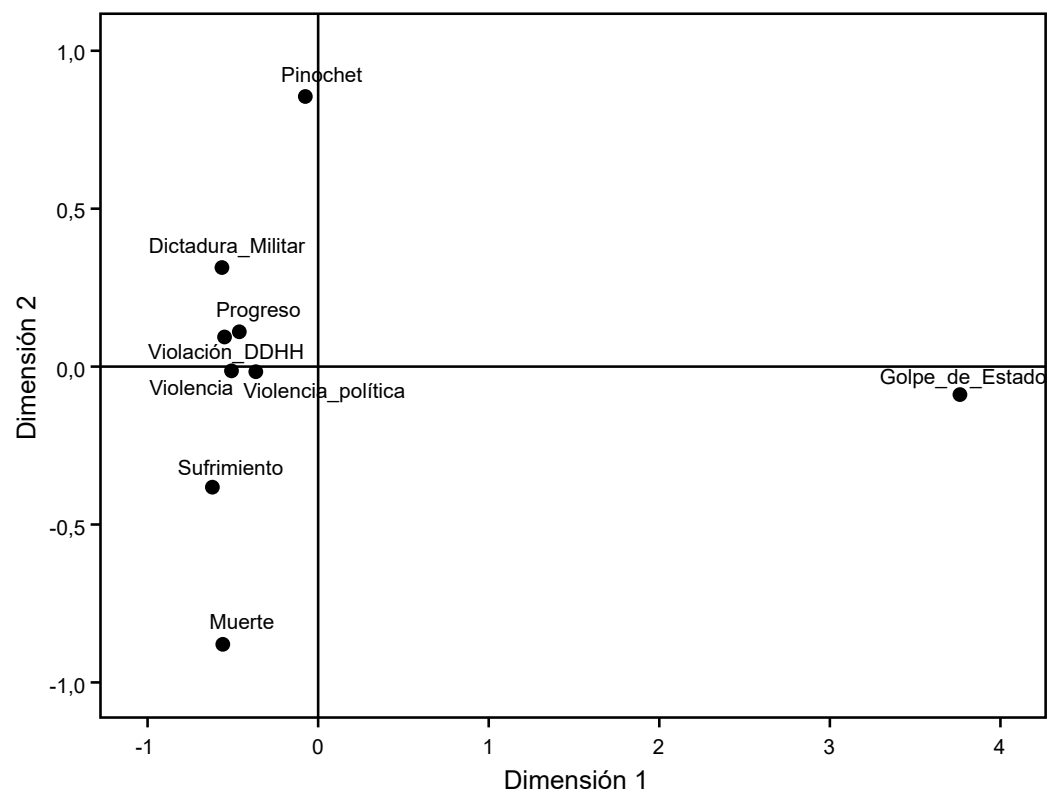

Figura 2. Escalamiento multidimensional al estímulo "11 de septiembre de 1973". 
aunque estos dos últimos términos se encuentran muy cercanos con todos los restantes conceptos. Sería una dimensión que opone el golpe de Estado con sus consecuencias más evidentes. La segunda dimensión opone la figura de Pinochet con muerte y sufrimiento, vinculando directamente el agente sindicado como responsable con las consecuencias percibidas de su acción.

Los análisis segmentados de la muestra mediante la variable sexo nos indican leves diferencias entre grupos referidas a la segunda dimensión, donde para el caso de los hombres se agregan a los conceptos ya enunciados los de dictadura militar y progreso. Para el caso de las mujeres, la figura de Pinochet desaparece de la dimensión oponiendo muerte con violación de derechos humanos (y junto a esta, violencia política y dictadura militar). Para el caso de los jóvenes que cursan niveles más bajos las diferencias se observan también en la segunda dimensión, donde la figura de Pinochet se opone a Sufrimiento, muerte y dictadura, siguiendo un patrón más parecido al de la muestra de hombres. Para el caso de los estudiantes de cursos más altos la dimensión dos opone muerte con dictadura militar, violación de derechos humanos y Pinochet.

Finalmente, se realizó un análisis de correspondencias múltiples (Figura 3) que en conjunto explican el $31.97 \%$ de la inercia (varianza). La primera dimensión opone el golpe de Estado con el sufrimiento y la violación de derechos humanos. La segunda dimensión opone a la figura de Pinochet, la dictadura militar y la violación de derechos humanos con la violencia. Es decir, nuevamente estamos frente a dimensiones que relevan las consecuencias negativas del hecho y que lo vinculan con el agente principal de dicha acción.

Los análisis realizados, segmentando la muestra mediante las variables sexo y nivel educativo, nos indican que no existen diferencias en las dimensiones resultantes del análisis de correspondencias en la asociación libre al estímulo 11 de septiembre de 1973, siendo las nociones de agente y consecuencia las que organizan sus juicios sobre el mismo. 


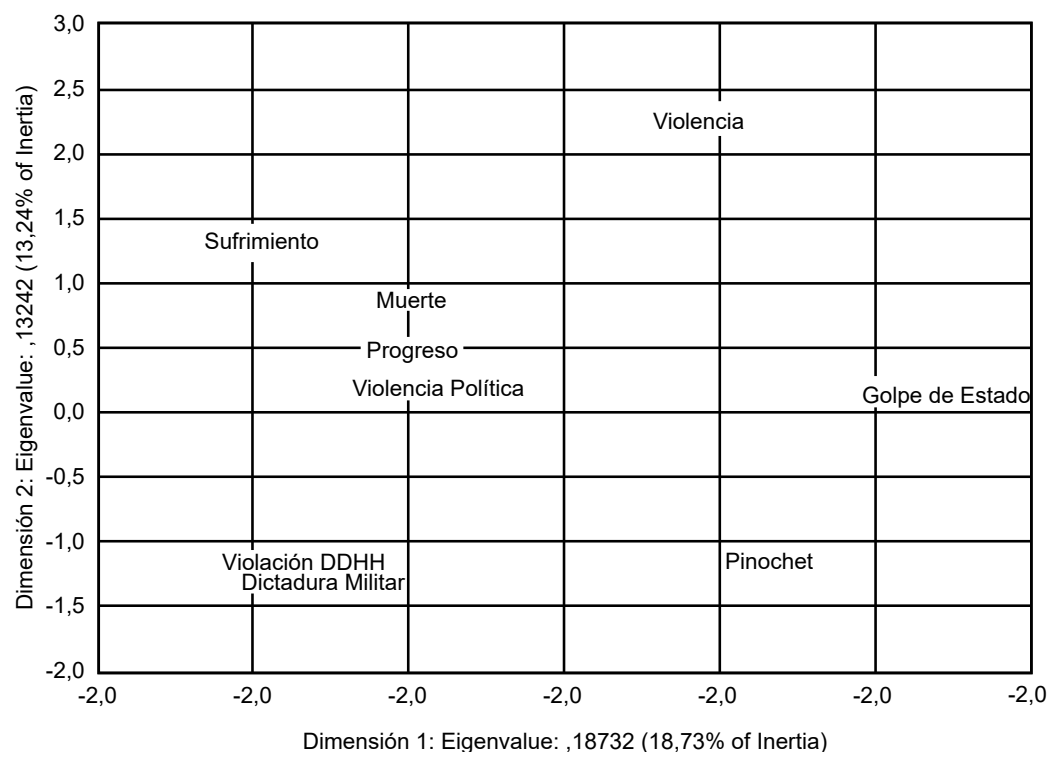

Figura 3. Análisis de correspondencias múltiples al estímulo

"11 de septiembre de 1973 "

\section{Discusión}

El objetivo de la presente investigación fue explorar en NNA chilenos/as las representaciones sociales del pasado, a nivel del campo de representación, específicamente sobre el 11 de septiembre de 1973, fecha del golpe de Estado en Chile. En términos generales, los resultados de este estudio proporcionan evidencia empírica de que los/ as NNA construyen una representación del golpe de Estado, asociada principalmente a sus efectos - muerte, violaciones de derechos humanos, sufrimiento, progreso, etc- $-\mathrm{y}$ a la figura de Pinochet como su principal agente.

Los resultados indican como primer hallazgo relevante que, casi la mitad de los participantes no logran asociar conceptos a la fecha señalada o asocian conceptos relativos a otros eventos históricos, tales 
como el atentado de las Torres Gemelas del 11 de septiembre de 2001 en Estados Unidos o la Primera Junta Nacional de Gobierno de Chile el año 1810, por mencionar algunos. Esto es llamativo, dado que el golpe de Estado es uno de los principales hitos de la historia política contemporánea chilena, y más de la mitad de los participantes no asocia concepto alguno a dicha fecha. Esto podría explicarse por varias causas.

En primer lugar, a la amplia despolitización experimentada por la sociedad chilena (Angelcos, 2011) y el desprestigio de la política (Valenzuela, 2011), lo que ha llevado a que las personas en general y los jóvenes en mayor medida se distancien de la política (Hatibovic \& Sandoval, 2015). Este fenómeno también, podría explicarse por la debilidad de las políticas de memoria en Chile. A nivel social aún no han otorgado suficiente reconocimiento a las víctimas de terrorismo de Estado en dictadura, cuestión que expresa sus efectos en la menor información con que cuentan los jóvenes, en relación con los adultos, sobre los distintos informes de las Comisiones de Verdad y Reconciliación que se han realizado en Chile (Arnoso et al., 2012). Una de las grandes tareas pendientes durante la posdictadura ha sido la educación y formación en Derechos Humanos, su inclusión en los planes y programas de estudio en todos los niveles formativos, así como un debate abierto sobre el período de violencia y sus efectos indirectos en la vida cotidiana de las personas aun transcurridos muchos años desde entonces.

La socialización política es otra dimensión que podría darnos algunas luces sobre este fenómeno. Si bien los estudios han planteado que la familia cumple un rol preponderante en la socialización política (Sandoval \& Hatibovic, 2010), sin embargo se habla muy poco de política con los/as NNA (Navarrete, 2008). Dicho déficit tiene su origen, probablemente, en el miedo que la dictadura dejó instalado en las relaciones sociales, en la idea que las diferencias políticas podían dividir irremediablemente a las familias (de allí que en Chile aún se utilice una frase que prohíbe hablar en la mesa familiar de política y religión). Del mismo modo, los planes y programas de estudio escolar han venido progresivamente reduciendo las horas destinadas a formación ciudadana (se retiró el antiguo ramo de educación cívica que, aún durante 
dictadura, persistía como materia fundamental de la formación escolar) (Mardones, 2018).

La ausencia de representaciones construidas por las nuevas generaciones sobre el golpe de Estado del 11 de septiembre de 1973 también da cuenta de las deudas que el sistema educativo y en particular el sistema político presentan para la socialización política en tanto valoración de la democracia y la política como espacio de encuentro con los otros distintos (Arendt, 1997; Palma, 2015). Si bien, en la actualidad la temática del Terrorismo de Estado y violación de los Derechos Humanos en Chile forma parte del curriculum escolar (Rojas \& Vargas, 2013), en la práctica, la enseńanza de esta queda a criterio del profesor de Historia. Como a muchos les resulta conflictivo enseñar este periodo prefieren no abordarlo o hacerlo desde su posición política personal (Toledo et al., 2015). Incluso en aquellos cursos donde se habla del período en cuestión este se hace desde una perspectiva demasiado amplia de derechos humanos y nunca llega a conversarse sobre las causas de la dictadura, sobre qué fue aquello que prohibió (el proyecto de la Unidad Popular) y cómo afecta a nuestra sociedad hasta al presente. La dinámica escolar de la sala de clases suele incluir las siguientes narración: el gobierno de la unidad popular hizo las cosas mal y generó una profunda división social; el golpe de Estado se tornó inevitable y aunque trajo dolor y sufrimiento, y por ello nunca más debe ocurrir, abrió el camino para el progreso social y económico de la nación (Palma, 2017).

A partir de los resultados del estudio, también es posible distinguir las representaciones sobre el 11 de septiembre de 1973, en específico los elementos del campo de representación, así como las dimensiones subyacentes que organizan dichas representaciones. En este sentido, los y las estudiantes distinguen las consecuencias negativas a nivel social y personal, pero también la creencia de un cierto progreso social — sobre todo en hombres-. Es decir, se reconocen los elementos negativos del golpe de Estado y posterior dictadura, pero se les vincularía también con un cierto progreso. Estas representaciones sobre el 11 de septiembre, nos sirven para interpretar el pasado y se constituyen en guías para la acción presente (Bobowik et al., 2014), cumpliendo una 
función de estabilidad social. Esto podría ser interpretado en el sentido de que se ha construido con mucha dificultad un espacio precario de estabilidad — “defendamos lo bueno detrás de lo malo”- , reforzando el valor social que ha tenido la estabilidad social y política en la historia de Chile (Jocelyn-Holt, 1998; Salazar \& Pinto, 1999).

Por otra parte, en relación con las dimensiones subyacentes, los resultados nos indican la presencia de dos dimensiones principales. La primera de ella referida a las consecuencias directas del golpe de Estado, tanto positivas como negativas. La segunda dimensión da cuenta del agente principal detrás golpe de Estado (Augusto Pinochet). En términos generales, estas representaciones confirman lo planteado por otros estudios, que dan cuenta que el golpe de Estado no es asociado a un proceso histórico complejo o de más largo alcance (Díaz et al., 2013). Estos resultados se podrían explicar por la distancia de estos(as) jóvenes con el hecho mismo (Carvacho et al., 2013; Reyes, 2007, 2015). Pero también es interesante destacar el hecho de que estas representaciones, al centrarse en el agente, ocultan el apoyo civil que recibió el golpe de Estado.

Durante el gobierno de la Unidad Popular, diversos actores - militares, empresarios, partidos políticos de derecha y de centro, entre otros - se "conjuraron" en contra del gobierno de la Unidad Popular (González, 2001), lo que sumado al rol de EE.UU. y la CIA (Kornbluh, 2013) generaron una poderosa alianza para derrocar al gobierno democrático. Sin embargo, esta parte de la historia es omitida por los y las estudiantes, visualizando a Pinochet como el principal agente causante del golpe. Esto podría ser explicado por la forma en que se desarrolló la transición chilena, donde la derrota de Pinochet en el Plebiscito de 1988, no significó la pérdida de poder, cuestión que se manifestó en que el carácter cívico-militar del golpe y la dictadura misma (Faúndez \& Azcárraga, 2014) se proyectó a la posdictadura (Jocelyn-Holt, 1998). En dicha etapa se aseguró la mantención de la Constitución Política de 1980, la protección de Pinochet y la no derogación de la Ley de Amnistía (Godoy, 1999). Las condiciones de posdictadura, impactaron y siguen impactando en las políticas y prácticas de educación asociada 
al golpe de Estado y posterior dictadura, ya que incluso palabras como "golpe de Estado" o "dictadura", durante muchos años fueron reemplazadas en los libros de historia por "pronunciamiento militar" o "régimen militar", respectivamente. En este sentido, sería interesante en futuros estudios analizar el modo en que se incorporan en el presente estos temas en el currículo escolar de educación primaria y secundaria.

Tal como se señaló anteriormente, es importante destacar que en Chile la mayor parte de los estudios sobre representaciones sociales del pasado traumático se han centrado en población adulta (Manzi, 1999; Manzi et al., 2003), por lo que este estudio contribuye con un conocimiento relevante sobre la población joven. En específico, los participantes del estudio pertenecen a una población expuesta a menores niveles de socialización política por habitar en una comuna de bajos ingresos económicos, con sectores rurales periféricos y un pequeño núcleo urbano.

El presente estudio no está exento de limitaciones. Si bien se utilizó un método que permite explorar conceptos que emergen de los propios participantes, pudiendo aproximarnos a una imagen del núcleo de las representaciones sociales del golpe de Estado en Chile, sería necesario complementar con otros instrumentos que permitan capturar la dimensión actitudinal e informacional de las representaciones sociales. Otra limitación del estudio consiste en que, al realizarse con una muestra perteneciente a una comuna específica de la región de Valparaíso, no es posible tener una perspectiva acabada del país, dada la diversidad geográfica y cultural de Chile.

Por último, este trabajo se constituye en una invitación a profundizar en la investigación y problematización sobre el modo en que los/as NNA construyen representaciones del pasado reciente y cómo éstas se traducen en visiones políticas del presente. Es relevante desarrollar estudios que busquen la superación de los discursos políticos que pretenden una reconciliación forzada y que sean reemplazados por discursos que apelen a una verdad plena de nuestra historia reciente. Del mismo modo, es importante señalar los desafíos que implica para futuras investigaciones trabajar con NNA como sujetos capaces de 
comprender reflexivamente su historia y trabajar por la ampliación de la democracia y los derechos colectivos. Estudiar cómo se representan el pasado implica pensar cómo viven su presente y cómo pueden llegar a proyectar su futuro. De allí la relevancia de estudios como estos y, sobre todo, en zonas rurales donde las políticas de la dictadura y posdictadura repercutieron de un modo distinto al de las grandes zonas urbanas.

\section{Referencias}

Abric, J-C. (1994). Les représentations sociales: Aspects théoriques. En J-C. Abric, Practiques sociales et represéntations. París: PUF. Angelcos, N. (2011). Elementos para una crítica de la despolitización en Chile. Revista Observatorio de Juventud 29(1), 64-84.

Arnoso, M., Cárdenas, M. \& Páez, D. (2012). Diferencias intergeneracionales en la mirada hacia el pasado represivo chileno. Psicología Politica, 45, 7-26.

Arnoso, M, Páez, D., Cárdenas, M., Zubieta, E., Espinosa, A. \& Bilbao, M. (2015). Representaciones sociales del pasado y rituales de justicia transicional en América Latina. Cuadernos de Pesquisa 45(156), 276-298. http://dx.doi.org/10.1590/198053143134

Banchs, M. (2000). Representaciones sociales, memoria social e identidad de género. Akademos, 2(1), 59-76.

Bobowik, M., Páez, D., Liu, J. H., Licata, L., Klein, O. \& Basabe, N. (2014). Victorious justifications and criticism of defeated: Involvement of nations in world wars, social development, cultural values, social representations of war, and willingness to fight. International journal of intercultural relations, 43, 60-73. https://doi.org/10.1016/j.ijintrel.2014.08.013

Cartes, C. (2014). Villa Grimaldi: reflexiones desde la pedagogía. El caso de los Colegios Polivalente York y Maipo. Tesis para optar al grado de licenciado en Historia, Universidad Academia de Humanismo Cristiano. 
Carvacho, H., Manzi, J., Haye, A., González, R. \& Cornejo, M. (2013). Consenso y disenso en la memoria histórica y en las actitudes hacia la reparación en tres generaciones de chilenos. Psykhe, 22(2), 33-47. http://dx.doi.org/10.7764/psykhe.22.2.601

Cárdenas, M. \& Blanco, A. (2004). Las representaciones sociales del movimiento antiglobalización. Psicología Politica, 28, 27-54.

Castro, C. (2013). Jóvenes secundarios y dictadura militar. Chile (1980-1989): Narrativas juveniles sobre la protesta social y la violencia politica, una aproximación desde la memoria social de estudiantes secundarios de Santiago. Tesis para optar al grado de licenciado en Educación, y al título de profesor de Historia y Ciencias Sociales. Santiago, Chile: Universidad Academia de Humanismo Cristiano.

Contreras, M.J., Grass, M. \& Nicholls, N. (2009). Pajarito nuevo la lleva. Teatro e memória: estratégias de representação e elaboração cênica da memória traumática infantil. Revista de Estudos de Literatura Artes e Culturas de Expressão Hispânica, 17, 154-172. https://doi.org/10.17851/2317-2096.17.0.154-172

Davoine, F. \& Gaudillière, J. M. (2011). Historia y trauma, La locura de las guerras. Buenos Aires: Fondo de Cultura Económica.

De Alba, M. (2016). Teorías en diálogo: representaciones sociales y memoria colectiva. Iztapalapa. Revista de Ciencias Sociales y Humanidades, 80(37), 131-151. https://doi.org/10.28928/ revistaiztapalapa/802016/aot1/dealbagonzalezm

Díaz, C., Faivovich, A., K., Carrasco, M., Glavic, K. \& Vivanco, S. (2013). Los ruidos del silencio. Los niños, niñas y adolescentes hablan a cuarenta años del golpe militar en Chile. Santiago: LOM Editores.

Di Giacomo, J. P. (1980). Intergroup alliances and rejections within a protest movement (analysis of the social representations). European Journal of Social Psychology, 10(4), 329-344. https:// doi.org/10.1002/ejsp.2420100402

Di Giacomo, J. P. (1987). Teoría y métodos de análisis de las representaciones sociales. En D. Páez (Ed.), Pensamiento, individuo y 
sociedad: cognición y representación Social (pp. 278-296). Madrid: Editorial Fundamentos.

Doise, W. (1991). Las representaciones sociales: presentación de un campo de investigación. Anthropos, suplementos, 27, 196-206.

Faúndez, X. \& Azcárraga, B. (2014). Aquí están: consecuencias familiares de la detención y desaparición forzada a cuarenta años del golpe de Estado en Chile. En Centro de Estudios Culturales Latinoamericanos. XIII Jornadas de Estudiantes de Postgrado en Humanidades, Artes y Ciencias Sociales. Universidad de Chile, Chile.

Faúndez, X., Cárdenas, M., Hatibovic, F., Palma, E. \& Bravo, D. (2017). Memories of Boys, Girls, and Adolescent Victims of Political Prison and Torture by the Chilean Military-Civilian Dictatorship. Journal of Interpersonal Violence, 1-27. https://doi. org/10.1177/0886260517721897

Faúndez, X., Cornejo, M. \& Brackelaire, J. L. (2013). Transgeneracionalidad del trauma psicosocial: imágenes de la detención de presos políticos de la dictadura militar chilena, Reconstruidas por los nietos. Psykhe, 22, 83-95. https://doi.org/10.7764/ psykhe.22.2.563

Faúndez, X., Cornejo, M. \& Brackelaire, J-L. (2014). Narration et silence: transmission transgénérationnelle du trauma psychosocial chez des petits-enfants de la dictature militaire chilienne. Cahiers de Psychologie Clinique, 43(2), 173-204. https://doi. org/10.3917/cpc.043.0173

Fundación OPCIÓN (2013). Los ruidos del silencio. Los niños, niñas y adolescentes hablan a cuarenta años del golpe militar en Chile. OPCION por los derechos de niñas y niños.

Godoy, O. (1999). La transición chilena a la democracia: pactada. Estudios Públicos, 74, 79-106.

González, M. (2001). Chile, la conjura: los mil y un dias del golpe. Santiago: Ediciones B.

Haas, V. \& Jodelet, D. (1999). Pensée et mémoire sociale. En J. Pétard (Coord.), Psychologie Sociale. París: Bréal Editions. 
Halbwachs, M. (1925). Les cadres sociaux de la mémoire. París: Albin Michel.

Halbwachs, M. (1950). La memoria colectiva. Zaragoza: Prensas Universitarias de Zaragoza.

Hatibovic, F. \& Sandoval, J. (2015). Una representación metafórica de la acción política en estudiantes de universidades chilenas. Ultima Década, 23(42), 11-37. http://dx.doi.org/10.4067/ S0718-22362015000100002

Hiner, H. (2015) ¿El "nunca más” tiene género? Un análisis comparativo de las comisiones de la verdad en Chile y Argentina. Estudios Sociales, 20, 253-270.

Jelin, E. (2002). Los trabajos de la memoria. Madrid, España: Siglo XXI. Jocelyn-Holt, A. (1998). El Chile perplejo. Santiago: Planeta/Ariel.

Jodelet, D. (1987). Representaciones sociales: un área en expansión. En D. Páez, C. San Juan, I. Romo y A. Vergara (Eds.), SIDA: imagen y prevención. Madrid: Fundamentos.

Jodelet, D. (2012). Conflits entre histoire memorielle et histoire historienne. Psicologia e Saber Social, 1(2),151-162. https://doi. org/10.12957/psi.saber.soc.2012.4896

Joya, C. A. C. (2006). ¿ ¿Nuevos o viejos debates? Las representaciones sociales y el desarrollo moderno de las ciencias sociales. Revista de Estudios Sociales, 25, 81-94.

Knapp, E., Suárez, M. \& Mesa, M. (2003). Aspectos teóricos y epistemológicos de la categoría representación social. Revista Cubana de Psicología, 20(1), 23-34.

Kornbluh, P. (2013). The Pinochet file: A declassified dossier on atrocity and accountability. New York: The New Press.

Le Paumier, F. \& Zavalloni, M. (2002). Mémoire collective et système identitaire: de Maurice Halbwachs à l'ego-écologie. En S. Laurens y N. Roussiau (Eds.), La mémoire sociale. Identités et représentations sociales. Rennes: PUR.

Liu, J. H. \& Hilton, D. J. (2005). How the pass weighs on the present: Social representations of history and their impact on identity politics. British Journal of Social Psychology, 44, 537-556. https:// doi.org/10.1348/014466605X27162 
Liu, J. H. \& Sibley, C. G. (2009). Culture, social representations, and peacemaking: A symbolic theory of history and identity. En C.J. Montiel, N.M. Noor (Eds.), Peace Psychology in Asia (pp. 21-39). https://doi.org/10.1007/978-1-4419-0143-9_2

Magendzo, S., Rubio, M. \& Aubel, B. (1999). Vivencia infantil del clima de violencia y represión bajo dictadura su relación con la constricción y el miedo, cuando jóvenes. Psykhe, 8(2), 73-83.

Manzi, J. (1999). Aportes de la psicología política al Chile Actual. Representaciones y actitudes acerca de la política en niños y jóvenes. En O. Corrales \& J. Sandoval (Eds.), Psicología politica. Cambio cultural y participación social (pp. 124-138). Valparaíso: Universidad de Valparaíso.

Manzi, J., Helsper, E., Ruiz, S., Krause, M. \& Kronmüller, E. (2003). El pasado que nos pesa: la memoria colectiva del 11 de septiembre de 1973. Revista de Ciencia Política, 23(2), 177-214. https://doi.org/10.4067/S0718-090X2003000200009

Mardones, R. (2018). Educación cívica y construcción de ciudadanía en el Chile de la pos dictadura, ¿en qué estamos y para dónde vamos? Revista Austral de Ciencias Sociales, 35, 63-82. https:// doi.org/10.4206/rev.austral.cienc.soc.2018.n35-04

Middelton, D. \& Edwards, D. (1990). Collective remerbering. Londres: Sage.

Moscovici, S. (1979). El psicoanálisis, su imagen y su público. Buenos Aires: Editorial Huemul.

Navarrete, B. (2008). Juventud y política en liceos municipales. El caso de Maipú. Última década, 16(28), 167-202. https://doi. org/10.4067/S0718-22362008000100009

Páez, D., Bobowik, M., Liu, J. \& Basabe, N. (2016). Shared beliefs about world history and cultural context: A theoretical review and a collective-level analysis. En C. Stone \& L. Bietti (Eds.), Contextualizing human memory: An interdisciplinary approach to urderstanding how individuals and groups remember the past (pp. 102-123). Nueva York: Routledge. https://doi. org/10.4324/9781315815398-7 
Palma, E. (2015). Profe ¿qué se celebra el 11 de septiembre? Memorias de niñas y niños sobre la dictadura militar chilena (1973-1990). Revista electrónica Perspectivas. Universidad de Chile.

Palma, E. (2017). Memorias de la dictadura chilena en la institución escolar. Un estudio de casos en escuelas primarias. Tesis para optar al grado de doctor en Ciencias Sociales, Facultad Latinoamericana de Ciencias Sociales, Argentina.

Reyes, M. (2007). El pasado reciente en el Chile de hoy: entre la reconciliación y la convivencia. Persona y sociedad, 21(1), 39-58.

Reyes, M. (2015). Construcción de políticas de memoria desde la vida cotidiana. Psicologia \& Sociedade, 27(2), 341-350. https://doi. org/10.1590/1807-03102015v27n2p341

Reyes, M. J., Cornejo, M., Cruz, M. A., Carrillo, C. \& Caviedes, P. (2015). Dialogía intergeneracional en la construcción de memorias acerca de la dictadura militar chilena. Universitas Psychologica, 14(1), 255-270. https://doi.org/10.11144/Javeriana.upsy14-1.dicm

Rojas, M. \& Vargas, D. (2013). La enseñanza del Golpe de Estado y la dictadura en Chile: Un diálogo entre historia y memoria. Cuaderno de Educación 55. Santiago de Chile: Universidad Alberto Hurtado. Recuperado de http://mailing.uahurtado.cl/ cuaderno_educacion_55/pdf/articulo55.pdf

Salazar, G. \& Pinto, J. (1999). Historia contemporánea de Chile Vol. I (Estado, legitimidad y ciudadanía). Santiago: Ediciones LOM.

Sandoval, J. (2004). Representación, discursividad y acción situada. Introducción critica a la psicología del conocimiento. Valparaíso, Chile: Universidad de Valparaíso.

Sandoval, J. \& Hatibovic, F. (2010). Socialización política y juventud: el caso de las trayectorias ciudadanas de los estudiantes universitarios de la región de Valparaíso. Ultima década, 18(32), 11-36. http://dx.doi.org/10.4067/S0718-22362010000100002

Toledo, M. \& Magendzo, A. (2013). Golpe de Estado y dictadura militar: estudio de un caso único de la enseñanza de un tema controversial en un sexto ańo básico de un colegio privado de la 
Región Metropolitana - Santiago, Chile. Psykhe, 22(2). http:// dx.doi.org/10.7764/psykhe.22.2.585

Toledo M., Magendzo, A., Gutiérrez, V., Iglesias, R. \& López-Facal, R. (2015). Enseñanza de "temas controversiales" en el curso de historia, desde la perspectiva de los estudiantes chilenos. Revista de Estudios Sociales, 52, 119-133. http://dx.doi.org/10.7440/ res52.2015.08.

Valenzuela, A. (2011). Crisis de representación y reforma politica en Chile. Documento presentado en el Seminario sobre reforma a los partidos. Santiago de Chile, Centro de Estudios Públicos (CEP) y Corporación de Estudios para América Latina (CIEPLAN).

Vásquez, G. (2014). Concepciones de los estudiantes chilenos de educación media sobre el proceso de transición de la dictadura a la democracia. Tesis para optar al Grado de Doctor en Didáctica. Valladolid: Universidad de Valladolid.

Veneros, D. \& Toledo, M (2009). Del uso pedagógico de lugares de memoria: visita de estudiantes de educación media al Parque por la Paz Villa Grimaldi (Santiago, Chile). Revista Estudios Pedagógicos, 35(1), 199-220. https://doi.org/10.4067/S071807052009000100012

Recibido: 23 de agosto, 2018

Revisado: 20 de marzo, 2019

Aceptado: 24 de julio, 2019 